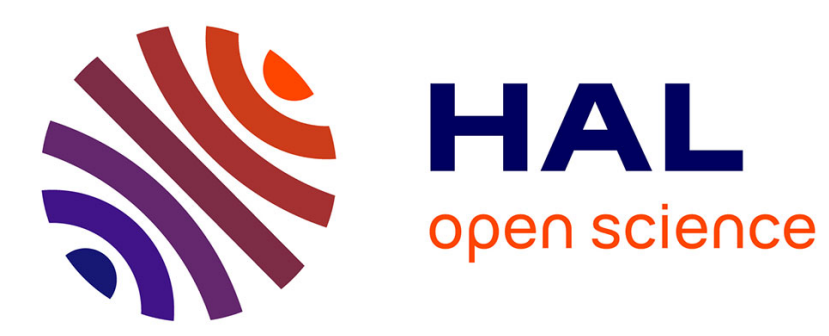

\title{
Hysteretic behavior induced by an electroacoustic feedback loop in a thermo-acousto-electric generator
}

Gaelle Poignand, C. Olivier, Guillaume Penelet, Pierrick Lotton

\section{To cite this version:}

Gaelle Poignand, C. Olivier, Guillaume Penelet, Pierrick Lotton. Hysteretic behavior induced by an electroacoustic feedback loop in a thermo-acousto-electric generator. Applied Acoustics, 2016, 105, pp.110 - 115. 10.1016/j.apacoust.2015.11.001 . hal-01400254

\section{HAL Id: hal-01400254 \\ https://hal.science/hal-01400254}

Submitted on 5 Mar 2019

HAL is a multi-disciplinary open access archive for the deposit and dissemination of scientific research documents, whether they are published or not. The documents may come from teaching and research institutions in France or abroad, or from public or private research centers.
L'archive ouverte pluridisciplinaire HAL, est destinée au dépôt et à la diffusion de documents scientifiques de niveau recherche, publiés ou non, émanant des établissements d'enseignement et de recherche français ou étrangers, des laboratoires publics ou privés. 


\title{
Hysteretic behavior induced by an electroacoustic feedback loop in a thermo-acousto-electric generator
}

\author{
G. Poignand, C. Olivier, G. Penelet, P. Lotton \\ Laboratoire d'Acoustique de l'Université du Maine, UMR CNRS 6613, Avenue Olivier \\ Messiaen, 72085 LE MANS Cedex 9, France.
}

\begin{abstract}
An active control method of the spatial distribution of the acoustic field is applied in a thermo-acousto-electric generator. An auxiliary acoustic source is used to force the self-sustained thermoacoustic oscillation in order to control the thermoacoustic amplification. The auxiliary source consists of a loudspeaker, located inside the loop-tube close to the main ambient heat exchanger, and supplied with a delayed signal through an electric feedback loop, comprising a phase-shifter and an amplifier, connected to a reference microphone. Experiments are performed on a prototype engine working with air at a static gauge pressure of 5 bars. Experimental results demonstrate how it is possible to tune the acoustic oscillations in order to increase the global performance of the generator, compared to the case without control, as well as the existence of a hysteretic behavior induced by the electroacoustic feedback loop itself, which leads to a discrepancy between the onset heat input and the offset one.

Keywords: Thermoacoustics, Heat engine, Acoustic field, Loudspeakers, Forced oscillations
\end{abstract}

\section{Introduction}

The thermoacoustic effect relies on the interaction between a porous material submitted to a temperature gradient and an oscillating gas, which leads to

Email address: gaelle.poignand@univ-lemans.fr (G. Poignand)

Preprint submitted to Elsevier

September 11, 2015 
the onset of self-sustained acoustic oscillations. Such an interaction can be used 5 to build thermoacoustic engines, a kind of piston-free thermodynamic engines which convert thermal power into acoustic power. In 1999, Backhaus and Swift [1] made a significant contribution by proposing a specific design of a thermoacoustic engine able to achieve a high thermoacoustic efficiency of $30 \%$ (41\% of Carnot efficiency). The geometry of this engine, a torus-shaped waveguide attached to a resonator, provides the required acoustic field in the porous material (referred to as the regenerator) to achieve a Stirling cycle. More recently, an engine with a similar design built by Tijani et al. [2] reached a record efficiency of $32 \%$ ( $49 \%$ of Carnot efficiency). Besides the possibility to achieve a high efficiency, thermoacoustic engines have a simple construction with no moving parts 15 and they usually operate with inert gases which are environmentally friendly. These advantages compared to conventional engines make them attractive in a wide variety of applications. In particular, a thermoacoustic Stirling engine can be coupled to an alternator in order to produce green electricity from waste heat recovery. In such a device, called a Thermo-Acousto-Electric Generator 20 (TAEG), the engine transforms the available heat into acoustic power which is further converted into electrical power via the alternator. Since the first TAEG built by Backhaus et al. [3] producing $60 \mathrm{~W}$ of electric power with an efficiency of $18 \%$, other prototypes have been developed to address various requirements, such as the decrease of the onset temperature [4] to meet the need for waste 25 heat recovery at low temperature, or the electricity production from a few watts with a low cost $[5,6]$, up to several $\mathrm{kW}[7]$ for energy recovery from industrial waste heat sources or solar source.

Tools [8] and methodologies [9] used to design or to predict the operation of thermoacoustic engines are based on the linear thermoacoustic theory estab30 lished by Rott [10]. However, although the linear theory can accurately predict the onset of the thermoacoustic instability [11], this theory fails to predict the acoustic wave saturation at high amplitudes, which is governed by complex nonlinear processes. These nonlinear processes can affect the engine operation by dissipating acoustic power (through cascade process of higher harmonics gen- 
eration $[12,13]$ or minor losses due to geometrical singularities $[14,15])$ or by modifying the temperature field distribution (through acoustically induced thermal conductivity [10] or acoustic streaming $[16,17,18,19,20])$. For this last mechanism, Penelet et al. [21] have shown that a change in the shape of the temperature field leads to a modification of the acoustic field which directly impacts

40 the thermoacoustic amplification process. Thus, above the onset and because of nonlinear effects, the temperature distribution (and therefore the acoustic field) deviates unavoidably from the one for which the engine was initially designed. A common solution to counteract these effects is to add passive elements such as membranes [22], jet pumps [1, 22] or tapered tubes [1, 23].

In this paper, our focus is rather oriented to investigate if there is a possibility to control the acoustic field in the thermoacoustic core in order to maximize the process of thermoacoustic amplification. For this purpose, an active control method is used to tune the spatial distribution of the acoustic field by means of an auxiliary acoustic source. This method comes down to drive the selfsustained thermoacoustic oscillation by an external source. Such experimental studies have already been carried out in thermoacoustics. Some studies have focused on the description of the variety of nonlinear dynamics behavior observed in experiments (synchronization $[24,25]$, route to chaos $[26], \ldots$ ). Other studies $[27,28,29,30,31]$ have focused on the forcing of acoustic oscillations in 55 the context of thermoacoustic engines. In particular, a proof-of-concept study provided by Desjouy et al. [28] in an annular engine has demonstrated that the addition of two auxiliary sources, appropriately tuned, can significantly improve the overall efficiency. More recently, Olivier et al. [31] successfully applied an active control method to the TAEG considered in this paper.

60

This study carries on with this latter work. The auxiliary source consists of a loudspeaker driven by an electric feedback loop, comprising a phase-shifter and an amplifier, connected to a reference microphone. The novelty lies in the choice of the auxiliary source position, which is located inside the loop-tube just over the main ambient heat exchanger. The aim of this study is to examine the effects of the active control of sound on the TAEG behavior. Experiments show 
two main results: the benefit of applying this method in terms of efficiency and temperature gradient at onset, and the existence of a hysteretic behavior leading to a discrepancy between the onset temperature and the offset temperature, caused by the feedback loop itself.

The experimental apparatus is briefly described in section II. The effects of the active control on the system behavior are then analyzed experimentally and compared to the case without active control. Finally, the last section focuses on further discussions about the application of active control feedback loop in thermoacoustic engines and future efforts to improve our understanding of the physical processes responsible for the observed phenomena.

\section{Experimental apparatus}

The experimental setup is a thermo-acousto-electric generator consisting of a Backhaus-Swift type thermoacoustic engine [1] coupled with an electrodynamic linear alternator, as shown in Fig. 1. It is filled with air at a gauge pressure of

805 bars. The main geometrical features of the set-up and and the fluid parameters are given in Table 1. The detailed description of the experimental apparatus can be found in ref. [31] and therefore only a brief description will be provided here.

The torus-shaped section contains a feedback waveguide (42 $\mathrm{mm}$ in diameter), a main ambient heat exchanger $\left(\mathrm{AHX}_{1}\right)$, a regenerator, a hot heat exchanger (HHX), a thermal buffer tube (TBT) and a secondary ambient heat exchanger $\left(\mathrm{AHX}_{2}\right)$. Its unwrapped length is $1.12 \mathrm{~m}$. The regenerator consists of a pile of stainless steel wire-mesh screens. Its porosity is about $69 \%$ and the hydraulic radius of the screen is $20 \mu \mathrm{m}$. The ambient heat exchangers are water-cooled to be kept at room temperature $T_{\mathrm{c}} \approx 293 \mathrm{~K}$, whereas the hot heat exchanger is heated at a hot temperature $T_{\mathrm{h}}$ by a Nichrome resistance ribbon. The inner wall of the part of the torus shaped waveguide (56 $\mathrm{mm}$ in diameter) comprised between the two ambient heat exchangers is covered by a ceramic tube in order to reduce the thermal leaks to the outside. The elements of the 


\begin{tabular}{|c|c|c|c|c|}
\hline \multicolumn{2}{|c|}{ Working fluid } & & & \\
\hline Fluid & Air & & & \\
\hline Static pressure & $0.5 \mathrm{MPa}$ & & & \\
\hline Ambiant temperature & $293 \mathrm{~K}$ & & & \\
\hline \multicolumn{5}{|c|}{ Torus Loop } \\
\hline Component & Length & Diameter & Porosity & Hydraulic radius \\
\hline Feedback waveguide & $915 \mathrm{~mm}$ & $42 \mathrm{~mm}$ & & \\
\hline Cone & $35 \mathrm{~mm}$ & & & \\
\hline Compliance & $30 \mathrm{~mm}$ & $56 \mathrm{~mm}$ & & \\
\hline $\mathrm{AHX}_{1}$ & $15 \mathrm{~mm}$ & $56 \mathrm{~mm}$ & 0.60 & $1 \mathrm{~mm}$ (cylindric pore) \\
\hline Regenerator & $23 \mathrm{~mm}$ & $56 \mathrm{~mm}$ & 0.85 & $0.05 \mathrm{~mm}$ (mesh grid) \\
\hline HHX & $15 \mathrm{~mm}$ & $56 \mathrm{~mm}$ & 0.89 & $0.30 \mathrm{~mm}$ (square pore) \\
\hline TBT & $50 \mathrm{~mm}$ & $56 \mathrm{~mm}$ & & \\
\hline $\mathrm{AHX}_{2}$ & $15 \mathrm{~mm}$ & $56 \mathrm{~mm}$ & 0.60 & $1 \mathrm{~mm}$ (cylindric pore) \\
\hline Jonction & $22 \mathrm{~mm}$ & $56 \mathrm{~mm}$ & & \\
\hline
\end{tabular}

Coupling waveguide

\begin{tabular}{lrr}
\hline Component & Length & Diameter \\
Waveguide & $1250 \mathrm{~mm}$ & $42 \mathrm{~mm}$ \\
Cone & $300 \mathrm{~mm}$ & \\
& &
\end{tabular}

Alternator back cavity $\quad 255 \mathrm{~mm} \quad 358 \mathrm{~mm}$

Table 1: Principal fluid and geometrical parameters of the thermoacoustic device. 


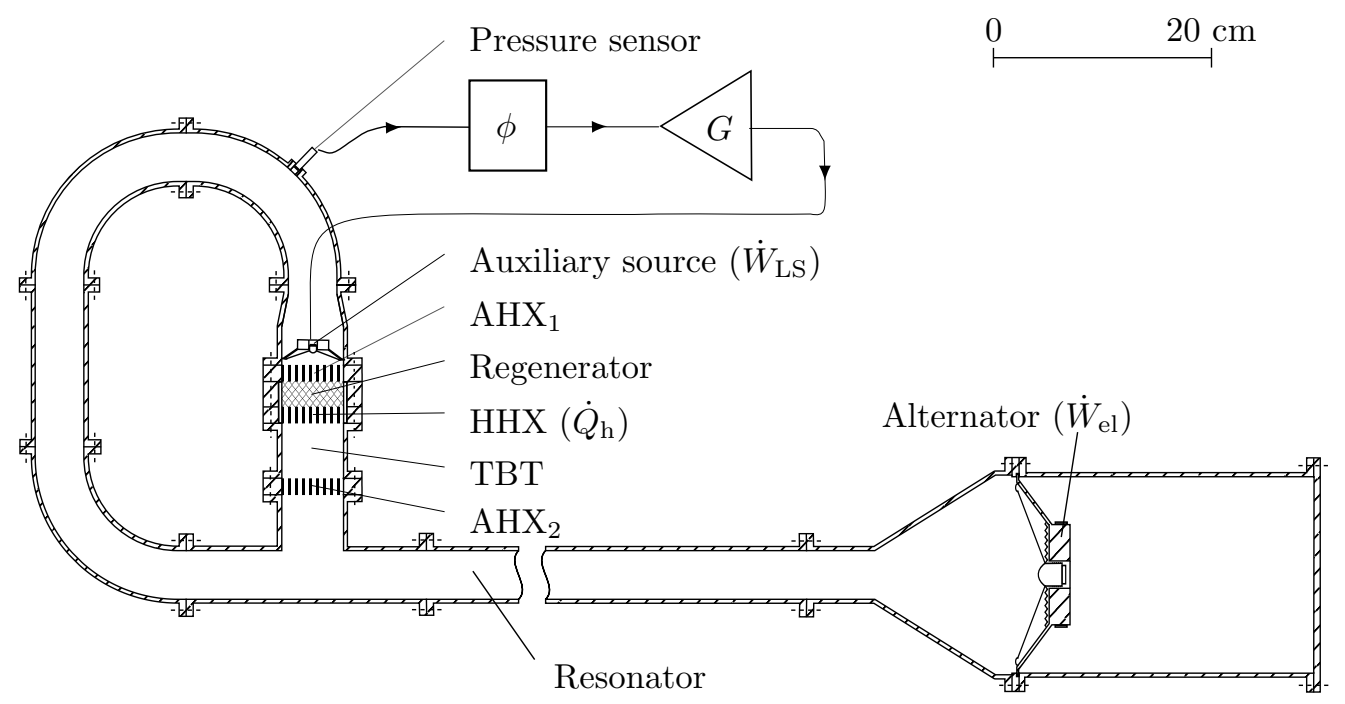

Figure 1: Schematic illustration of the thermo-acousto-electric generator.

torus loop are designed to realize the traveling-wave phasing with high acoustic impedance in the regenerator, as required for a high efficiency thermoacoustic amplification. The torus section is connected via a coupling waveguide $(1.55 \mathrm{~m}$ in length and $42 \mathrm{~mm}$ in diameter) to the alternator and its rear cavity (6.3 l in volume). The alternator is a classical electrodynamic loudspeaker (Monacor SPH-170C). A variable resistor is used to load electrically the alternator in order to extract electrical power $\dot{W}_{\mathrm{el}}$.

The active control system, used to drive the autonomous oscillations, consists of an auxiliary acoustic source, namely an electrodynamic loudspeaker (Aura NSW2-326-8A), powered by the output signal from a pressure sensor $\mu$ via the electroacoustic feedback loop which includes a phase-shifter and an audio amplifier. The phase-shifter consists of three all-pass filter in series. Thus, the acoustic source input signal is the pressure sensor signal phase-shifted with a phase-shift $\phi$ and amplified with a voltage gain $G$. The novelty here compared to the previous study [31] lies in the acoustic source position: it is located inside the loop-tube, whereas it was enclosed in a small cavity and coupled to the loop guide via a capillary tube in the previous study. It is placed just above 
the main ambient heat exchanger at a high acoustic impedance position (i.e. large acoustic pressure and low volume velocity), which is suitable for the low displacement of the auxiliary source (its maximal excursion is $\pm 3 \mathrm{~mm}$ ). Furthermore, the auxiliary source introduces an acoustic load in the engine, whose effect is to increase the heat input which is necessary to trigger the onset of selfsustained oscillations (even when no power is supplied to the auxiliary source), but a model used to describe the onset threshold [11] shows that the heat input rise is the lowest at this position.

All the measurements are done in the steady state regime, which means that between each measurement a time delay is respected until temperature stabilization in the whole device. In order to monitor the temperature, thermocouples are flush-mounted along the device. In particular, two thermocouples measure the temperature difference $\Delta T$ between the temperature $T_{\mathrm{h}}$ in the center of the hot heat exchanger and the temperature $T_{\mathrm{c}}$ in the center of the ambient heat exchanger. A piezoelectric pressure sensor, schematically shown in Fig. 1, measures the acoustic pressure above the main ambient heat exchanger. The input heat power $\dot{Q}_{\mathrm{h}}$ dissipated by Joule effect into the ribbon of the hot heat exchanger is delivered by a DC-power supply (Aim-TTi EX 4210R). The input electric power $\dot{W}_{\mathrm{LS}}$ supplied to the auxiliary loudspeaker is obtained from the measurement of both the voltage difference and the current (via an AC current probe) passing through the moving coil. Note that the AC current probe was preliminarily calibrated by measuring the current through a $7 \mathrm{Ohm}$ resistor at different frequencies, especially in order to prevent from any source of error related to a phase shift inherent to the probe itself. The maximum input electric power $\dot{W}_{\mathrm{LS}}$ value is $\mathbf{1 5}$ W. The outgoing electrical power $\dot{W}_{\text {el }}$ is obtained from the measurement of the voltage difference across the load resistor. The ratio of the outgoing to incoming electrical powers gives the overall efficiency of the engine:

$$
\eta=\frac{\dot{W}_{\mathrm{el}}}{\dot{Q}_{\mathrm{h}}+\dot{W}_{\mathrm{LS}}} .
$$



resistance). 

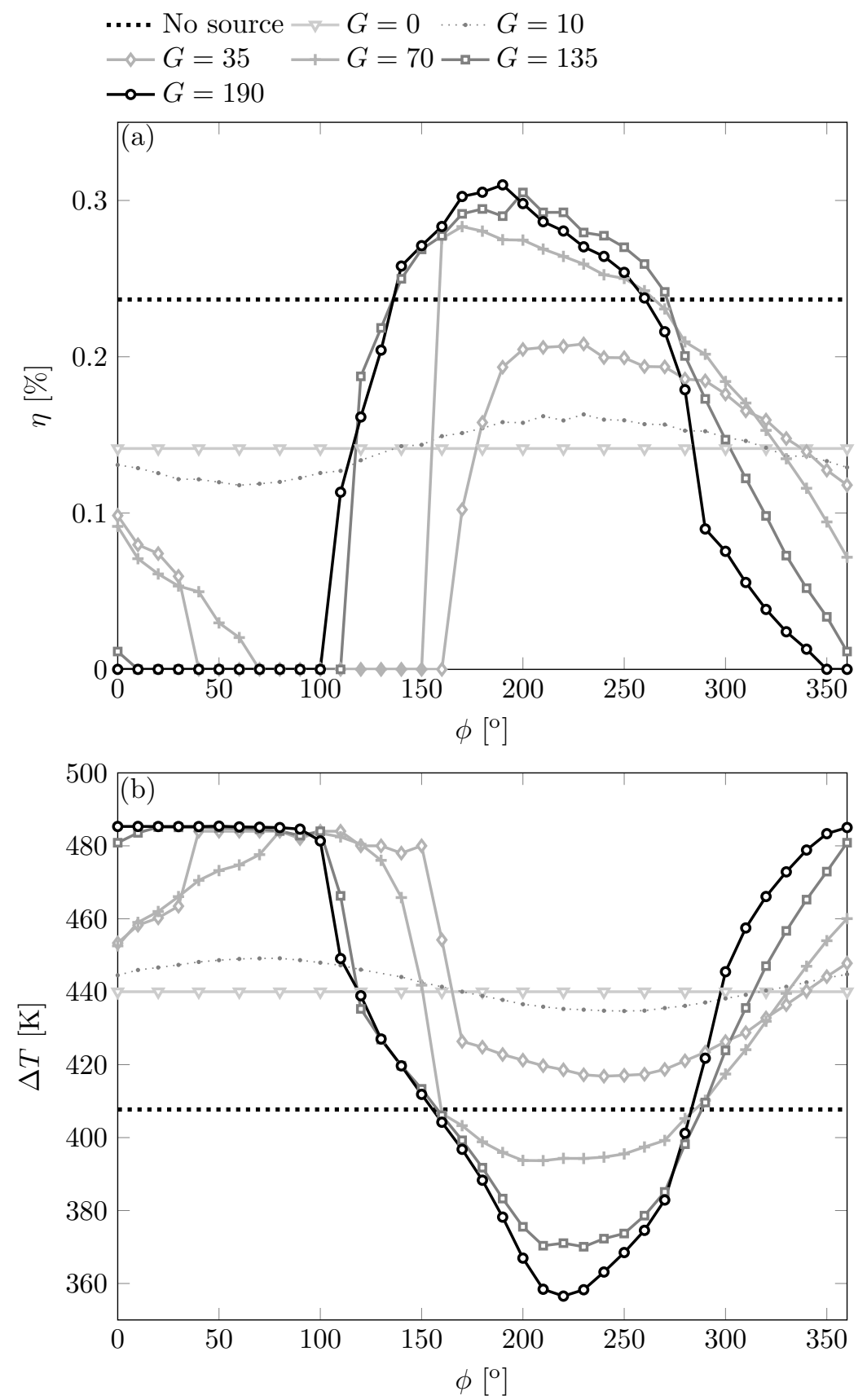

Figure 2: (a) Efficiency $\eta$ as a function of the imposed phase-shift $\phi$ for different voltage gains $G$ at fixed heat power $Q_{\mathrm{h}}$. (b) Corresponding evolution of the temperature difference $\Delta T$ for different voltage gains $G$. The dotted line corresponds to the initial engine without active control (that is to say, without any source introduced within the closed-loop). 


\subsection{Effect of phase-shift $\phi$ for different voltage gains $G$ at fixed heat power $Q_{\mathrm{h}}$}

We first look at the influence of the imposed phase-shift $\phi$ on the engine bedecrease below the one without active control. For example, at the maximum efficiency the temperature difference $\Delta T=356 \mathrm{~K}$ is $52 \mathrm{~K}$ lower than for the one 
corresponding to the initial engine.

It is also worth noting that there exists an "unfavorable" range of value for the imposed phase-shift $\phi$ which even leads to the quenching of self-sustained oscillations (e.g. for $\phi \in\left[-10^{\circ}: 100^{\circ}\right]$ at $G=35$ and for $\phi \in\left[70^{\circ}: 150^{\circ}\right]$ at $G=190$ ). In this latter case, the temperature difference $\Delta T$ is only controlled by heat diffusion through the thermoacoustic core (no sound, and therefore no heat transport by sound) and it rises to a higher value than in the case when thermoacoustic conversion occurs. Lastly, the phase-shift domain for which the acoustic wave is suppressed extends with the voltage gain.

\subsection{Effect of voltage gains $G$ for two heat powers $Q_{\mathrm{h}}$ at fixed phase-shift $\phi$}

To complete these results, experiments are conducted at a constant phaseshift $\phi$, when the voltage gain $G$ varies, for two different heat powers: $Q_{\mathrm{h}}=70 \mathrm{~W}$ and $100 \mathrm{~W}$, respectively just above and away from the onset heat input for $G=0$ $\left(Q_{\mathrm{h}, \text { onset }}=68 \mathrm{~W}\right)$. The phase-shift $\phi$ is fixed at a value included in the optimal phase range, $\phi=200^{\circ}$ and $220^{\circ}$ respectively for $Q_{\mathrm{h}}=70 \mathrm{~W}$ and $100 \mathrm{~W}$. Fig. 3 presents the efficiency $\eta$ and the temperature differences $\Delta T$ evolutions with the voltage gain $G$. As previously observed, for a phase-shift included in the optimal phase range, the gain increase leads to a gradual efficiency increase and a gradual temperature difference decrease. The active control has an interest for both heat powers considered, since the efficiency $\eta$ increases (by about $30 \%$ for $Q_{\mathrm{h}}=70 \mathrm{~W}$ and $9 \%$ for $100 \mathrm{~W}$ ) and the temperature difference $\Delta T$ decreases (by about $53 \mathrm{~K}$ for $Q_{\mathrm{h}}=70 \mathrm{~W}$ and $8 \mathrm{~K}$ for $100 \mathrm{~W}$ ) compared to the case without active control (dotted line). However, the impact of the active control is less important for a higher heat input. Moreover, the increase of efficiency saturates when increasing the voltage gain $G$. In fact, for a high gain or a high input power, the electric power supplied to the auxiliary source becomes significant (up to 7.6 W at $G=212$ and $Q_{\mathrm{h}}=70 \mathrm{~W}$ ) and hence comes to limit the overall efficiency of the engine. 

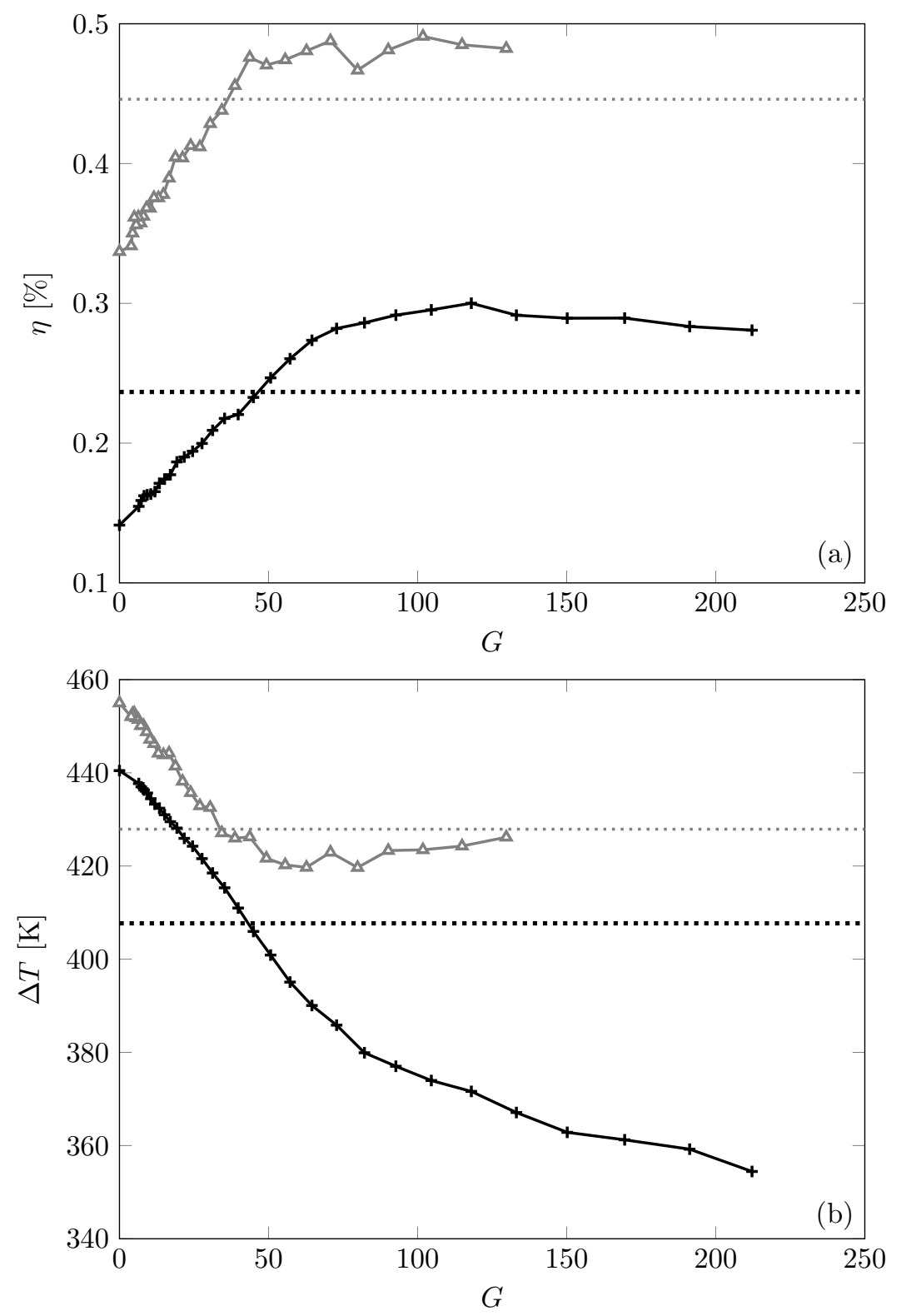

Figure 3: (a) Efficiency $\eta$ as a function of the voltage gain $G$ for two heat powers $Q_{\mathrm{h}}=70 \mathrm{~W}$ $(+)$ and $100 \mathrm{~W}(\triangle)$ at a fixed phase-shift $\phi$, respectively $200^{\circ}$ and $220^{\circ}$. (b) Corresponding evolution of the temperature difference $\Delta T$. The dotted line corresponds to the initial engine without active control (that is to say, without any source introduced within the closed-loop). 


\subsection{Effect of heat power $Q_{\mathrm{h}}$ for different voltage gains $G$ at fixed phase-shift $\phi$}

The next results concern the observation of the onset and offset of the therAt the same time, the offset temperature difference decreases as well from $437 \mathrm{~K}$ to $320 \mathrm{~K}$, in such a way that the area of the hysteresis loop increases, meaning 

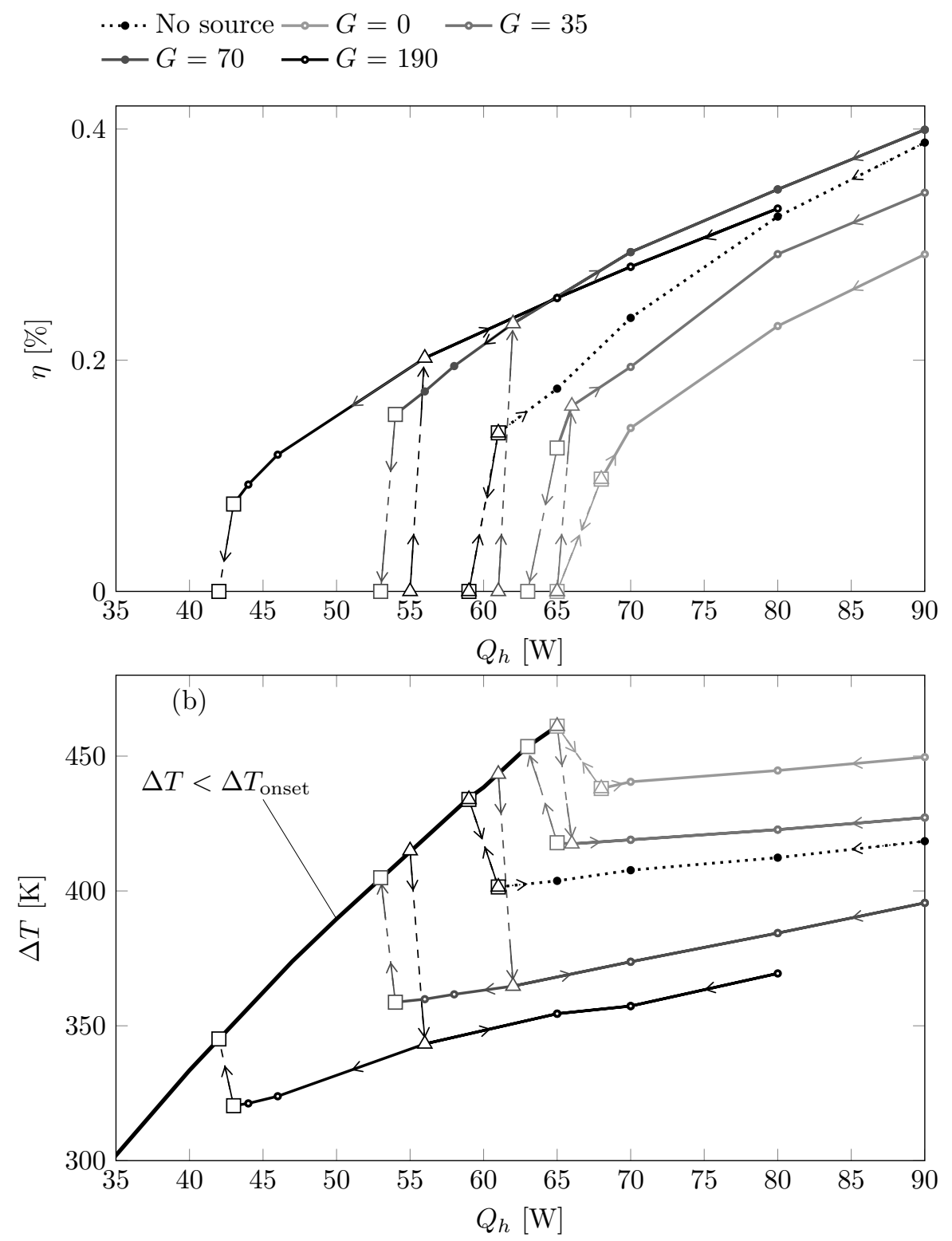

Figure 4: (a) Hysteretic behavior of the efficiency $\eta$ with the heat input $Q_{\mathrm{h}}$, at a fixed phase-shift $\phi=220^{\circ}$ and for different voltage gains $G$. (b) Corresponding evolution of the temperature difference $\Delta T$ for different voltage gains $G$. The black line corresponds to the temperature above the onset of thermoacoustic instability. The dotted line corresponds to the initial engine without active control. Arrows indicate the direction of hysteresis. The triangles indicate onset conditions and the squares offset conditions. 
that the working temperature range extends toward lower temperatures. This lower temperatures.

\section{Discussion}

This study was conducted to investigate the effect of an active control method on the behavior of a TAEG. This method consists in forcing the selfsustained thermoacoustic instability with a loudspeaker placed inside the looptube in order to control the thermoacoustic amplification. The experimental results highlight that when the auxiliary source is appropriately tuned, some improvements are achieved in terms of efficiency and temperature difference across the regenerator, compared to the case without active control. These observations are consistent with the previous findings [31] observed in a similar setup. However, a major difference comes from the source position, located here inside the loop-tube. At this position, effects of the active control method on the system performance are enhanced in comparison with the previous study, since the efficiency increase reaches at the maximum $30 \%$ with a decrease of the temperature difference of $53 \mathrm{~K}$. Besides, it is also advantageous to place the auxiliary source inside the loop-tube since this suppresses the so-called Gedeon streaming, a second order time-average mass flow running clockwise in the engine (accordingly with Fig.1), which induces an undesired heat flux from the hot heat exchanger to the second ambient heat exchanger $\left(\mathrm{AHX}_{2}\right)$. Moreover, a major outcome in comparison with the previous study is the observation of a discrepancy between the onset and the offset temperature which causes a hysteretic behavior of both the temperature difference across the regenerator and the efficiency versus the heat input. As a result, the operating temperature range extends to the lower temperatures. This is an interesting result in the context of waste heat recovery, since this method may offer the possibility to recover heat at a lower temperature and thereby may open up new areas of application. 
Although these results seem promising, we have no clear idea at the present time about how the active control affects the engine behavior. One may interpret the results obtained simply by applying the superposition principle, the acoustic power radiated by the auxiliary source being just amplified by the thermoacoustic amplification core. We believe that such an analysis is not sufficient because it could neither explain the presence of quenching nor the hysteretic behavior induced by the electroacoustic feedback loop. Another possibility (hypothesis?) 295 is an indirect modification of the spatial distribution of the temperature field within the thermoacoustic core produced by a change of the acoustic nonlinear effects (for example streaming pattern).

Further studies need to be carried out to get a better understanding of the active control process. The development of a simplified analytic model would provide useful insight to describe the coupling between the thermoacoustic engine and the active control feedback loop and to determine how the active control method operates. This model would also provide a guidance for the selection of an appropriate auxiliary source in the early stage of engine design (in this study, the auxiliary source has been chosen by convenience and a posteriori). Moreover, this study has been performed on a TAEG academic model and the next step will be to experience this active control method in a high power TAEG, which is under construction in our laboratory.

[1] S. Backhaus, G. W. Swift, A thermoacoustic-stirling heat engine: Detailed study, J. Acoust. Soc. Am. 107 (6) (2000) 3148.

[2] M. E. H. Tijani, S. Spoelstra, A high performance thermoacoustic engine, J. Appl. Phys. 110 (9) (2011) 093519-093519-6.

[3] S. Backhaus, E. Tward, M. Petach, Traveling-wave thermoacoustic electric generator, Appl. Phys. Lett. 85 (6) (2004) 1085.

[4] K. De Blok, Novel 4-stage traveling wave thermoacoustic power genera- 
tor, in: Proceedings of ASME 2010 3rd joint US-European FEDSM2010ICNMM2010, American Society of Mechanical Engineers, 2010.

[5] Z. Yu, P. Saechan, A. J. Jaworski, A method of characterising performance of audio loudspeakers for linear alternator applications in low-cost thermoacoustic electricity generators, Appl. Acoust. 72 (5) (2011) 260-267.

[6] D. Zhao, Y. Chew, Energy harvesting from a convection-driven rijke-zhao thermoacoustic engine, J. Appl. Phys. 112 (11) (2012) 114507.

[7] Z. Wu, G. Yu, L. Zhang, W. Dai, E. Luo, Development of a $3 \mathrm{~kW}$ doubleacting thermoacoustic stirling electric generator, Appl. Energ. 136 (2014) 866-872.

[8] W. C. Ward, G. W. Swift, J. P. Clark, Interactive analysis, design, and teaching for thermoacoustics using DeltaEC, J. Acoust. Soc. Am. 123 (5) (2008) 3546 .

[9] A. C. Trapp, F. Zink, O. A. Prokopyev, L. Schaefer, Thermoacoustic heat engine modeling and design optimization, Appl. Therm. Eng. 31 (14-15) (2011) 2518-2528.

[10] N. Rott, Thermally driven acoustic oscillations, part III: Second-order heat flux, Z. Angew. Math. Phys. 26 (1) (1975) 43-49.

[11] M. Guedra, G. Penelet, On the use of a complex frequency for the description of thermoacoustic engines, Acta Acust. United Ac. 98 (2) (2012) $232-241$.

[12] H. Yuan, S. Karpov, A. Prosperetti, A simplified model for linear and nonlinear processes in thermoacoustic prime movers. part ii. nonlinear oscillations, J. Acoust. Soc. Am. 102 (6) (1997) 3497-3506.

340 [13] V. Gusev, H. Bailliet, P. Lotton, M. Bruneau, Asymptotic theory of nonlinear acoustic waves in a thermoacoustic prime-mover, Acta Acust. United Ac. 86 (1) (2000) 25-38. 
[14] G. Swift, A. S. of America, Thermoacoustics: A Unifying Perspective for Some Engines and Refrigerators, AIP Conference Proceedings / Atomic, Molecular, Chemical Physics Series, Acoustical Society of America through the American Institute of Physics, 2002.

[15] A. Berson, P. Blanc-Benon, Nonperiodicity of the flow within the gap of a thermoacoustic couple at high amplitudes, J. Acoust. Soc. Am. 122 (4) (2007) EL122-EL127.

[16] J. W. Strutt, On the circulation of air observed in kundt's tubes, and on some allied acoustical problems, Philos. Trans. R. Soc. Lond. 175 (1884) $1-21$.

[17] D. Gedeon, Dc gas flows in stirling and pulse tube cryocoolers, in: J. Ross, R.G. (Ed.), Cryocoolers 9, Springer US, 1997, pp. 385-392.

[18] G. P. Smith, R. Raspet, R. Hiller, J. McDaniel, Evanescent modes and anomalous streaming in a thermoacoustic device, Appl. Acoust. 69 (1) (2008) 23-30.

[19] G. Penelet, M. Guedra, V. Gusev, T. Devaux, Simplified account of rayleigh streaming for the description of nonlinear processes leading to steady state sound in thermoacoustic engines, Int. J. Heat Mass Transfer 55 (2122) (2012) $6042-6053$.

[20] I. Reyt, V. Daru, H. Bailliet, S. Moreau, J.-C. Valire, D. Baltean-Carls, C. Weisman, Fast acoustic streaming in standing waves: Generation of an additional outer streaming cell, J. Acoust. Soc. Am. 134 (3) (2013) 17911801.

[21] G. Penelet, S. Job, V. Gusev, P. Lotton, M. Bruneau, Dependence of sound amplification on temperature distribution in annular thermoacoustic engines, Acta Acust. United Ac. 91 (3) (2005) 567-577.

[22] G. W. Swift, D. L. Gardner, S. Backhaus, Acoustic recovery of lost power in pulse tube refrigerators, J. Acoust. Soc. Am. 105 (2) (1999) 711. 
[23] J. R. Olson, G. W. Swift, Acoustic streaming in pulse tube refrigerators: tapered pulse tubes, Cryogenics 37 (12) (1997) 769-776.

[24] G. Penelet, T. Biwa, Synchronization of a thermoacoustic oscillator by an external sound source, Am. J. Phys. 81 (4) (2013) 290-297.

[25] T. Yoshida, T. Yazaki, Y. Ueda, T. Biwa, Forced synchronization of periodic oscillations in a gas column: Where is the power source?, J. Phys. Soc. Jpn. 82 (10) (2013) 103001.

[26] T. Yazaki, Experimental observation of thermoacoustic turbulence and universal properties at the quasiperiodic transition to chaos, Phys. Rev. E 48 (1993) 1806-1818.

[27] P. S. Spoor, G. W. Swift, The huygens entrainment phenomenon and thermoacoustic engines, J. Acoust. Soc. Am. 108 (2) (2000) 588-599.

[28] C. Desjouy, G. Penelet, P. Lotton, Active control of thermoacoustic amplification in an annular engine, J. Appl. Phys. 108 (11) (2010) 114904$114904-7$.

[29] N. Pan, C. Shen, S. Wang, Experimental study on forced thermoacoustic oscillation driven by loudspeaker, Energ. Convers. and Manage. 65 (0) (2013) 84-91.

[30] S. Li, D. Zhao, Heat flux and acoustic power in a convection-driven t-shaped thermoacoustic system, Energ. Convers. and Manage. 75 (2013) 336-347.

[31] C. Olivier, G. Penelet, G. Poignand, P. Lotton, Active control of thermoacoustic amplification in a thermo-acousto-electric engine, J. Appl. Phys. 115 (17) (2014) 174905. 